\title{
Exploiting the dynamic properties of FET-based chemical sensors
}

\author{
P Bergveld \\ University of Twente, Box 217, 7500 AE Enschede, The \\ Netherlands
}

Abstract. After a long period of mainly static application of ISFETS, other more sophisticated applications are now being developed, based on the exploitation of the dynamic properties of ISFETS. Examples are the use of flow-through cells with sample injection and the integration of a $\mathrm{pH}$ actuator electrode for very fast titration in a microvolume. Also, the most recent development of an immunofet makes use of induced transient phenomena.

\section{Introduction}

In the early ISFET publications, dealing mainly with $\mathrm{pH}$ sensitive devices, this new type of sensor has always been compared with the well known glass-membrane $\mathrm{pH}$ electrode, because of the $\mathrm{SiO}_{2}$ layer in contact with an aqueous solution. The initial assumption was that the mechanism of ISFET operation should be similar to that of the glassmembrane electrode: at the surface a gel layer should exist, which exchanges $\mathrm{H}^{+}$ions with the aqueous solution, resulting in a diffusion potential according to the Nernst theory. The sensitivity of an ISFET was therefore expected to be the same as for a glass-membrane electrode (i.e. Nernstian) predicting $58 \mathrm{mV} / \mathrm{pH}$. If this was not observed, as was the case with $\mathrm{SiO}_{2}$ and $\mathrm{Si}_{3} \mathrm{~N}_{4}$, it was seen as an unexplained deviation from the theoretical value. Since about 1980, however, it has been recognised that the existing models in the colloid chemistry literature, which describe the oxide-electrolyte interfacial potential, were also applicable to ISFET devices, the most relevant model being the site-dissociation model. It could be proven that even with relatively simple theory, using parameters taken from the site-dissociation and double-layer models, experimental data from devices having surfaces with widely different reactive properties could be interpreted $[1,2]$. Application of inorganic layers such as $\mathrm{Al}_{2} \mathrm{O}_{3}$ and $\mathrm{Ta}_{2} \mathrm{O}_{5}$ deposited on the original $\mathrm{SiO}_{2}$ gate material improved the $\mathrm{pH}$ sensitivity of the devices, which could be fully explained theoretically.

Although the operational mechanisms of ISFETS and glass membrane electrodes - surface site dissociation and ion exchange respectively - are thus quite different, the original idea of their similarity is still maintained. This, however, is not unrealistic, because the non-Nernstian response of an ISFET is in fact adapted, in the related theory, by multiplying a Nernstian response by a dimensionless sensitivity parameter smaller than unity (see equation (6)). Furthermore it is striking that modern theories concerning the explanation of the glass-membrane electrode response make use of the sitedissociation theory [3] applied to reactive sites which are not only located at the surface, but also inside a surface gel layer.

Concerning their applications as well as the theoretical aspects, ISFETS were seen as a replacement for the glass- membrane electrode in those cases in which the latter sensors could not be used, as for instance with in vivo measurements of the chemical constituents of blood [4]. In that case it is absolutely essential that the sensor be as small as possible and very rigid, properties inherent to the modern silicon sensors to which class the ISFET belongs.

Although the fundamental behaviour of the oxideelectrolyte and the glass-electrolyte interfaces, in the case of an ISFET and a glass-membrane electrode, respectively, are now fully understood and can be described theoretically in relation to each other as mentioned above, the way of measuring the corresponding interfacial potentials in each case differs, however, to a large extent, as will be explained in the following section. This difference will be shown to be essential to the explanation of the observed difference in stability between ISFETS and glass-membrane electrodes.

\section{Basic ISFET theory}

In order to compare the modes of operation of ISFETS and glass-membrane electrodes in an actual measuring set-up, the basic ISFET theory should first be considered.

The ISFET can be seen as a special type of MOSFET, with the gate at a certain distance. Therefore we have to start the operational description with the basic MOSFET theory.

Figure 1 shows a schematic representation of an n-channel metal oxide semiconductor field effect transistor (MOSFET). It consists of two n-type diffusions, the source (S) and the drain (D), in a p-type silicon substrate. The structure is covered with an insulating layer of silicon dioxide on top of which a metal gate electrode $(G)$ is deposited over the area between source and drain. When a positive voltage is applied to the gate with respect to the source, electrons (which are the minority carriers in the substrate) are attracted to the surface and create a conducting channel between source and drain.

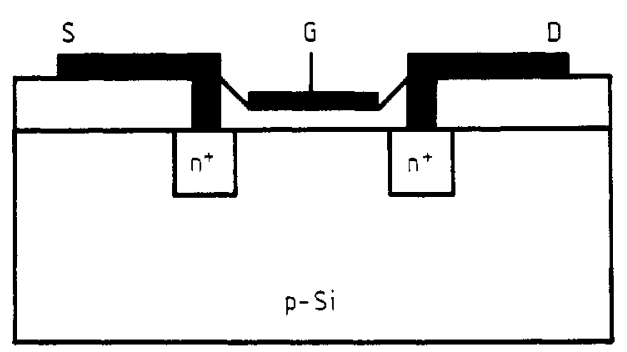

Figure 1. Schematic representation of a MOSFET device.

The conductivity of this channel can thus be modulated by the strength of the electric field that exists in the gate insulator between the metal gate electrode and the silicon. Therefore, the device is called a metal oxide semiconductor field effect transistor or MOSFET.

In the first-order mos transistor theory, the equation for the drain current, $I_{D}$, in the unsaturated region 
$\left(V_{\mathrm{D}}<V_{\mathrm{G}}-V_{\mathrm{T}}\right)$ is

$$
I_{\mathrm{D}}=\mu C_{\mathrm{ox}} \frac{W}{L}\left[\left(V_{\mathrm{G}}-V_{\mathrm{T}}\right) V_{\mathrm{D}}-\frac{1}{2} V_{\mathrm{D}}^{2}\right]
$$

where $\mu$ is the electron mobility in the channel, $C_{0 x}$ the capacitance per unit area of the oxide, $W / L$ the ratio of channel width to length, $V_{\mathrm{G}}$ and $V_{\mathrm{D}}$ the applied gate-source and drain-source voltages respectively and $V_{\mathrm{T}}$ the threshold voltage, which can be described as follows:

$$
V_{\mathrm{T}}=V_{\mathrm{FB}}-\frac{Q_{\mathrm{B}}}{C_{\mathrm{ox}}}+2 \varphi_{\mathrm{F}}
$$

where $Q_{\mathrm{B}}$ is the bulk depletion charge per unit area, $\varphi_{\mathrm{F}}$ is the Fermi potential difference between the doped bulk silicon and intrinsic silicon and $V_{\mathrm{FB}}$ is the flatband voltage given by

$$
V_{\mathrm{FB}}=\frac{\Phi_{\mathrm{M}}}{q}-\frac{\Phi_{\mathrm{Si}}}{q}-\frac{Q_{\mathrm{it}}+Q_{\mathrm{f}}}{C_{\mathrm{ox}}}
$$

where $\Phi_{M}$ and $\Phi_{\mathrm{Si}}$ are the metal and silicon work functions respectively, while $Q_{\mathrm{it}}$ and $Q_{\mathrm{f}}$ are the trapped interface charge and fixed oxide charge respectively, both per unit area.

In the first instance, the operation of a mos device in the unsaturated region can be explained fully by these three equations. In the saturated region $\left(V_{\mathrm{D}}>V_{\mathrm{G}}-V_{\mathrm{T}}\right)$, equations (2) and (3) are still valid, but equation (1) has to be replaced by

$$
I_{\mathrm{D}}=\frac{1}{2} \mu C_{\mathrm{ox}} \frac{W}{L}\left(V_{\mathrm{G}}-V_{\mathrm{T}}\right)^{2}
$$

The sensitivity of FET-based chemical sensors can be explained by the chemical modulation of one or more of the distinguishable parameters in these equations, as will be discussed below in the case of an ISFET.

As already mentioned, the ISFET can be seen as a special type of MOSFET, but with the gate at a certain distance. The ISFETS first developed and most frequently described are of the $\mathrm{pH}$-sensitive type. With these devices, the gate metal of the original MOSFET is simply omitted and replaced by an electrolyte solution which is contacted by a reference electrode. This is shown schematically in figure 2 .

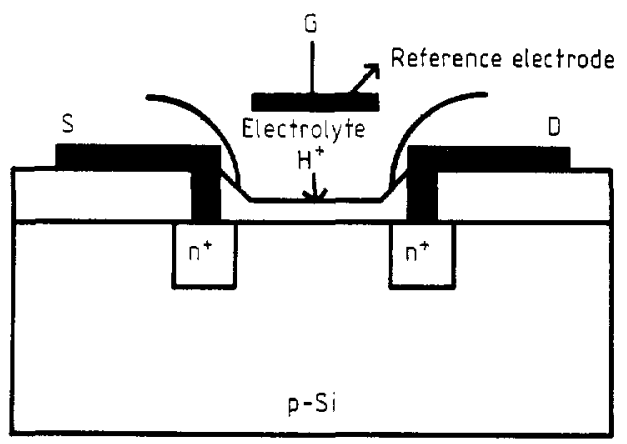

Figure 2. Schematic representation of an ISFET device.

The metal part of this reference electrode can be considered as the gate of a MOSFET, and consequently the original MOSFET equations can still be applied. However, in this case the flatband voltage $V_{\mathrm{FB}}$ as given in equation (3) changes into $[1,2]$

$$
V_{\mathrm{FB}}=E_{\mathrm{ref}}-\psi_{0}+\chi^{\mathrm{sol}}-\frac{\Phi_{\mathrm{Si}}}{q}-\frac{Q_{\mathrm{it}}+Q_{\mathrm{f}}}{C_{\mathrm{ox}}}
$$

In this equation, the chemical sensitivity of the ISFET is included; $E_{\text {ref }}$ is the absolute reference electrode potential and $\left(-\psi_{0}+\chi^{\text {sol }}\right)$ is the the potential that develops over the electrolyte-insulator interface. The surface dipole potential of the solvent, $\chi^{\text {sol }}$, can be considered to be constant. The surface potential $\psi_{0}$ depends on the $\mathrm{pH}$ of the solution.

The $\mathrm{pH}$ dependence of $\psi_{0}$ forms the actual basis for the chemical sensitivity of the ISFET and can be described by the site-dissociation model $[1,2]$ as mentioned in the previous section, resulting in the following equation:

$$
\psi_{0}=2.3 \frac{k T}{q}\left(\frac{\beta}{\beta+1}\right)\left(\mathrm{pH}_{\mathrm{pzc}}-\mathrm{pH}\right)
$$

under the condition that $\beta \gg q \psi_{0} / k T$. In this equation, $\mathrm{pH}_{\mathrm{pzc}}$ is the $\mathrm{pH}$ of the solution at which no net surface charge exists (so-called point of zero charge). $\beta$ is a parameter that reflects the chemical sensitivity of the gate insulator, depending on the density of hydroxyl groups and the surface reactivity. With this model, the sensitivities that are found for commonly used insulators like $\mathrm{SiO}_{2}, \mathrm{Al}_{2} \mathrm{O}_{3}$ and $\mathrm{Ta}_{2} \mathrm{O}_{5}$ can be explained excellently.

\section{The glass-membrane electrode}

As already mentioned in $\S 1$, the potential at the glasselectrolyte interface shows pure Nernstian behaviour, as described by the following equation:

$$
\psi_{0}=C+2.3 \frac{R T}{F} \mathrm{pH}
$$

In contrast with the ISFET, because the inner side of the glass membrane is contacted with an electrolyte, an additional interfacial potential, $\psi_{0}^{\prime}$, occurs, also obeying equation (7). This is drawn schematically in figure 3.

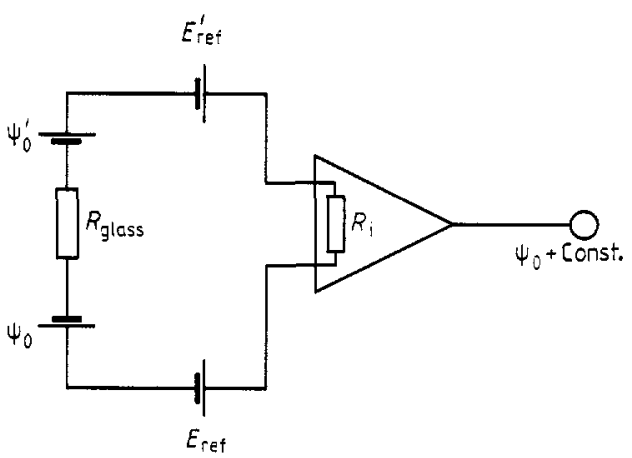

Figure 3. Schematic representation of the measuring circuit for the glass-membrane electrode.

Besides an external reference electrode as applied in the case of an ISFET, an internal reference electrode is now also applied, resulting in two series voltages, $E_{\text {ref }}$ and $E_{\text {ref }}^{\prime}$, in the measuring circuit. If the internal resistance of the electrometer amplifier, $R_{\mathrm{i}}$, is very large with respect to the bulk resistance $R_{\text {glass }}$ of the glass membrane, the output of the amplifier will accurately reflect $\psi_{0}(\mathrm{pH})$ with respect to the $\mathrm{pH}$ of the internal electrolyte (usually $\mathrm{pH} 7$ ), assuming that $E_{\text {ref }}=E_{\text {ref }}^{\prime}$.

\section{Stability of ISFETS versus stability of glass-membrane electrodes}

Comparing an ISFET and a glass-membrane electrode, we note that both devices measure $\psi_{0}$, even with the same sensitivity for high values of $\beta$ in the case of an ISFET. In this respect the 
two devices do not differ at all. However, a large difference manifests itself with respect to the offset.

While in the case of a glass-membrane electrode only a small offset occurs due to a slight asymmetry between the inner and outer glass surfaces as well as the reference electrodes, equation (5) shows that the ISFET exhibits a large 'offset', related to the physical constants in the value of the flatband voltage. These constants depend on the specific manufacturing process of MOSFETS and ISFETS. This means that in the gate dielectric, certain charges are present which are not necessarily immobile. Because an electric field is always present in the gate oxide, a small displacement of these charges may occur, resulting in a certain amount of device drift. Moreover, in the case of an ISFET with two dielectric layers $\left(\mathrm{SiO}_{2}\right.$ and $\left.\mathrm{Al}_{2} \mathrm{O}_{3}\right)$, additional charges may set up at the interface between these two insulators, while it is also reported that top layers such as $\mathrm{Al}_{2} \mathrm{O}_{3}$ show a certain polarisation, which will also result in a long-term drift [5]. These effects are typical for ISFETS and related devices, while they do not exist in glass-membrane electrodes because in the bulk of the glass membrane, no electric field is present, due to its conducting properties. This means that no space charges can be present in the glass and thus no drift phenomena can be expected. Therefore glass-membrane electrodes, as such, will definitely show better long-term stability than ISFETS. Considering the amount of research that has already been done to improve the drift behaviour of ISFETS, it is not very likely that their stability will approach that of glass-membrane electrodes in the very near future. However, their having such small dimensions combined with a very fast response means that ISFETS are very useful candidates for many chemical-sensor applications, for which various methods have been developed to decrease the effect of the intrinsic ISFET drift, as will be described in the following sections.

\section{Compensation of ISFET drift}

Experiments with $\mathrm{Al}_{2} \mathrm{O}_{3}$ ISFETS show that the drift rate stabilises after about 30 minutes of contact with an analyte and can be estimated as a linear function of time for at least the next $20 \mathrm{~h} \mathrm{[6].} \mathrm{It} \mathrm{is} \mathrm{found} \mathrm{that} \mathrm{the} \mathrm{origin} \mathrm{of} \mathrm{this} \mathrm{drift} \mathrm{is} \mathrm{a} \mathrm{solid-state}$ effect, located within the $\mathrm{Al}_{2} \mathrm{O}_{3}$ layer, because it is also present in MAOSFET test devices made in the same wafer as the ISFETS. This means that the drift of a certain batch of ISFETS can be predicted from the behaviour of the test devices, the value of which can, for instance, be stored in a PROM which is an integral part of the ISFET lead connector. The signal conditioner to which a particular ISFET is later connected

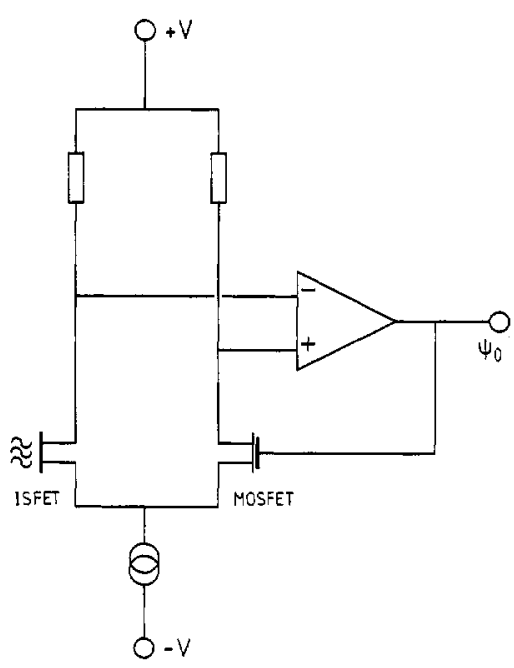

Figure 4. Diagram of the ISFET-MOSFET amplifier circuit. reads the predetermined drift behaviour and compensates for it. This procedure has been used with the Sentron catheter-tip $\mathrm{pH}$ electrode [4], in which case the stability of the measurement could be guaranteed within $0.02 \mathrm{pH}$ unit during $10 \mathrm{~h}$ of continuous in vivo measurement.

It may be expected, adapting the process parameters during the deposition of the $\mathrm{Al}_{2} \mathrm{O}_{3}$ layer, that the drift behaviour of the ISFETs can be better fixed to a predetermined value, making it possible to use the compensation method as mentioned above even for much longer periods of time. It should be noted, however, that the application of a compensation technique relies fully on the invariability of the quantity to be compensated, which in general cannot be said of exponentional functions, by their nature. Therefore it cannot be expected that the procedure of drift compensation would be a definite solution to drift problems of ISFETS in long-term applications, such as for implantable use.

\section{Applications of FET-based sensors}

\subsection{Application of ISFET-MOSFET pairs}

In the 'operational transducer' developed by the Newcastle upon Tyne ISFET group [7], an ISFET is integrated with a MOSFET as well as with a complete feedback amplifier which controls the gate voltage of the MOSFET in such a way that it tracks the change in $\psi_{0}$ of the ISFET. The system is drawn schematically in figure 4 .

In this way the temperature sensitivity as well as the drift of the ISFET is compensated for, as these quantities are the same for ISFET and MOSFET. In this case one has to rely on the ability to perfectly match the parameters of both devices, such as the threshold voltages and their stability, which is, however, not yet possible with the present NMOs processes in use for the fabrication of isfETs. Therefore, this approach is not expected to be a definite solution to the drift problem.

\subsection{Application of ISFET-REFET pairs}

A similar approach to that mentioned above is the application of a second ISFET made insensitive to ions, called REFET, connected in a differential circuit with the ISFET to be used for ion sensing [8]. This system is drawn schematically in figure 5 .

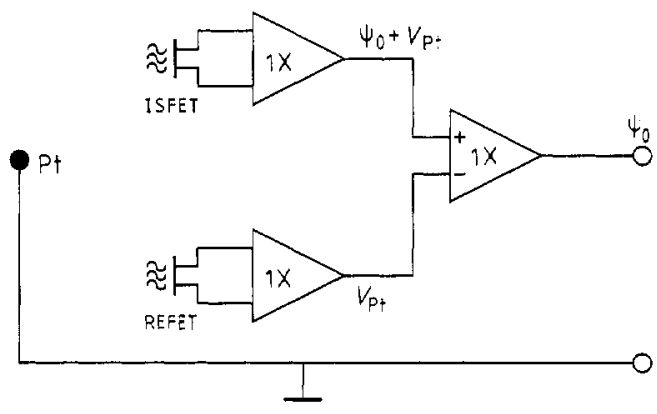

Figure 5. Diagram of the ISFET-REFET measuring system.

Although the main reason for this development is the replacement of a liquid-filled reference electrode by a pseudo-reference electrode ( $\mathrm{Pt}$ wire), made possible by the differential set-up, an additional feature would be the inherent compensation of equal deviations in both devices, such as the temperature-induced variations as well as the drift. Here, however, the same limitations as in the case of an ISFETMOSFET pair show up. The system will operate better than a single device, but it cannot be expected that the compensation will be perfect for a particular couple, because of the 
inability to match FETS perfectly with respect to stable threshold voltages.

\subsection{Alternative methods}

It should be noted that in all the systems described above, the ISFETS are explored for continuous and static applications. The drift problem therefore manifests itself strongly. The idea arises whether a sampling technique would not be more appropriate, whereby the response of an ISFET is determined alternately with respect to a carrier electrolyte and a sample of the analyte. In such systems the excellent dynamic properties of ISFETS and their small dimensions and planar structure are a great advantage, implying the ability to make very fast measurements in microlitre volumes. The following examples will show the validity of this approach in the application of tiny flow-through cells, microlitre titrations and the development of a novel immunofET.

\subsection{Flow-through cells with incorporated ISFETS}

The most sophisticated flow-through cell with integrated ISFETS and an analyte chopping analysis system has been developed by Sibbald and Covington [9], for which a schematic diagram is shown in figure 6.

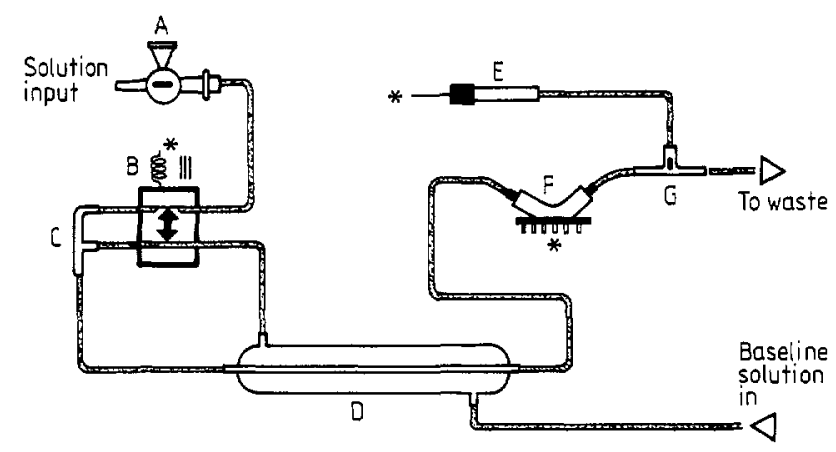

Figure 6. Schematic representation of a flow-through system. A, three-way Luer input tap; B, pinch-tube solenoid valve; C, Pyrex F-piece; D, Pyrex heat-exchanger; E, reference electrode, F, quadruple-function chemFET analysis cell; $G$, porous plug reference junction. (Reproduced with permission; Med. Biol. Eng. Comp.)

The aim of this development was the construction of an on-line blood-ion analyser as an alternative to catheter-tip sensors which have the disadvantage of intracorporeal usage. The flow cell contains one chip with four ISFETs, each having a particular membrane for selective measurement of one specific ion. The ISFET responses are measured with respect to a conventional type of (micro)reference electrode, placed at the output of the flow cell. Blood samples are sent alternately with a calibration solution to the flow cell, which is inserted in the carrier solution by means of a valve mechanism. The design considerations of the whole system have been governed, more or less, by the specific blood-related complications and not by the reduction of drift problems as such.

In view of the considerable physiological variations in physical and (bio)chemical properties of blood as well as the unknown effect of the addition of anticoagulants, the sample length in the tubing of the system is chosen such that the ISFET as well as the reference electrode in contact with the blood sample are in electrochemical equilibrium at the time of measurement. This means that it is the equilibrium potential and not the transient potential, as is usual with FIA systems, that is used as the signal of interest. The sampling period is therefore at least $25 \mathrm{~s}$.
A similar but more straightforward approach is applied in the microcell system developed by Gumbrecht et al [10], as represented schematically in figure 7 .

This microcell contains two identical ISFETs. The cell is connected to a double-lumen catheter with a slightly recessed inner lumen. Both ISFETS can be measured with respect to a pseudo-reference electrode ( $\mathrm{Pt}$ wire) as in the ISFET-REFET differential set-up as described in the previous section. To calibrate the ISFETS, a calibration solution is pumped through
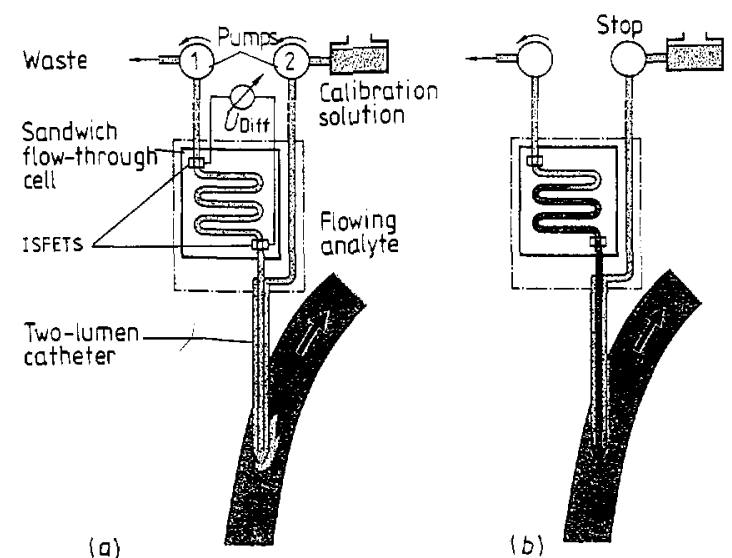

Figure 7. Schematic representation of a flow-through system. (a) Calibration mode: both ISFETs in contact with calibration solution. (b) Measurement mode: one ISFET in contact with analyte and the second in contact with the calibration solution.

the outer lumen of the catheter into the bloodstream. With a second pump a fraction of this solution is withdrawn from the tip volume near the inner lumen and drawn into the microcell. This situation is illustrated in figure $7(a)$. The ISFETs can now be set (or reset) to the same baseline. After that, the flow direction in the outer lumen is reversed for some seconds, resulting in an uptake of blood into the cell as illustrated in figure $7(b)$. Now one of the ISFETS is in contact with the analyte, while the other is still in contact with the baseline solution, resulting in a differential signal. Next, both ISFETS come into contact with the analyte and the differential signal should again be zero. Finally, the second ISFET comes into contact with the analyte, while the first is again in contact with the calibration liquid by changing the flow direction in the outer lumen. The time delay between the calibration and the actual measurement is only $15 \mathrm{~s}$, remarkably reducing drift effects.

Note that the two chemical sensors are identical (both ISFETS) and do not differ in their chemical response as in the case of an ISFFET-REFET pair as described previously. This is essential for the reset procedure.

\subsection{ISFETS applied as a null detector}

Although the measurement system as a whole is much more complicated than the semi-FIA system as mentioned in the previous section, Tamura et al [11] developed a $\mathrm{pH}$ monitoring system in which the actual application of the ISFET, as such, is much simpler than in any other system. The ISFET is used only as a null detector, eliminating the drift problem completely, and the sensitivity of the ISFET is then not important. The system makes use of a servo control loop, which allows the equilibration between blood and a reference solution blended from two standard buffer solutions, as shown schematically in figure 8 . 


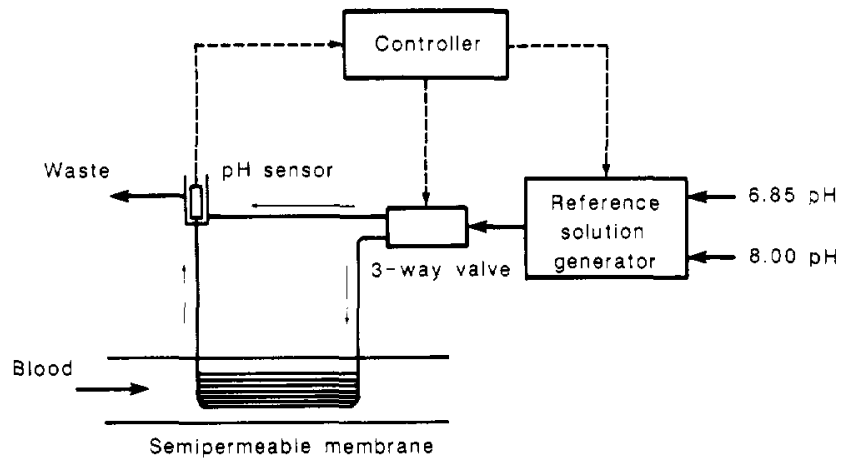

Figure 8. Schematic representation of equilibration system, in which the ISFET operates as a null detector. The servo control is operated by the ISFET output so as to reduce the difference in $\mathrm{pH}$ values of the blended reference solution, alternately directly and after contact with blood via a semipermeable membrane. Signal line, ...-; fluid line, - (Reproduced with permission; Sens. Actuators).

The blood is able to change the $\mathrm{pH}$ of the reference solution via a membrane, the result of which is measured by the ISFET in an alternate cycle with the original reference solution. A measured difference activates the servo control to adapt the $\mathrm{pH}$ of the reference solution in such a way that both measurements result in the same $\mathrm{pH}$ reading. This is the $\mathrm{pH}$ of the blood. Due to the fast response of ISFETs, the measurement cycle can be as short as $8 \mathrm{~s}$.

\subsection{ISFETS applied as an indicator electrode}

The examples mentioned in the previous sections were all devoted to the monitoring of blood $\mathrm{pH}$. In the sequence of examples, the actual application of the ISFET progressed from an equilibrium measurement over a short period to a transient measurement and finally only a null-detector function. A recent development in quite another field of application is found in the integration of a $\mathrm{pH}$-sensitive ISFET and a $\mathrm{pH}$ actuator, resulting in the development of a microlitre titrator [12] of which a cross section is shown in figure 9.

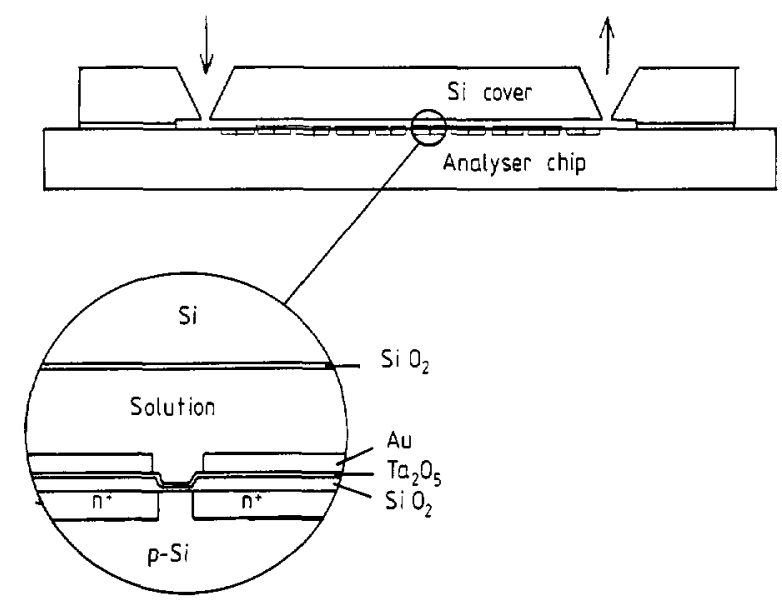

Figure 9. Cross section of a coulometric analyser. The solution layer is $30-100 \mu \mathrm{m}$ thick.

The titrator vessel consists of a cavity of depth $80 \mu \mathrm{m}$ etched into a silicon cover, which is sealed on an array of ten ISFETS. Each ISFET gate area is surrounded by a thin-film gold electrode. The application of a current between two of these electrodes will result in the electrolysis of water if the cavity is filled with an electrolyte. The $\mathrm{pH}$ will thus be locally increased at one electrode and decreased at the counter electrode, both $\delta \mathrm{pHs}$ being measured by the corresponding ISFETS. At one ISFET the $\delta \mathrm{pH}$ is measured with respect to a third ISFET which is not positioned in the titrated area, using one of the free gold electrodes as a pseudo-reference electrode. In this way it appears to be possible to titrate an acid or a base within a few seconds; the ISFET is only used as a very fast $\mathrm{pH}$-indicator electrode to determine the equivalence point of the coulometrically controlled titration.

Note that the signal of interest is no longer the value of a voltage but the value of an elapsed time after the onset of the coulometric current, which occurs within seconds, with chosen cell dimensions. Therefore drift problems no longer exist, while in addition the specific sensitivity of the ISFETS is no longer important.

Based on the experimental results achieved with the micro titrator as described above, a $\mathrm{CO}_{2}$ sensor with internal titration has also been developed [13], as well as a dipstick titrator [14].

\subsection{Immuno-modified ISFETS with equilibrium disturbance}

It has long been debated whether it would be possible to distinguish between the characteristic charge of an antibody/ antigen complex covering an ISFET and the charge of one of the separate constituents. This appears not to be possible using the immuno-modified FET (IMFET) in a static way. This problem is not caused directly by the ISFET drift behaviour, but is due to the porous structure of the membrane, including the proteins, which, moreover, are surrounded by neutralising counter ions, thus shielding any electric dipole that may exist and be modulated by a specific immunoreaction.

The existence of a porous membrane containing immobilised proteins with fixed charges and mobile counter charges can, however, be exploited using dynamic measurements [15]. If, at a certain $\mathrm{pH}$ differing from the isoelectric point of the protein (complex) immobilised in a membrane which covers the ISFET, a sudden change in the ionic concentration of the electrolyte is created, a temporary disturbance of the membrane potential will occur. This is the result of the effective mobility differences of cations and anions, determined by the value of the fixed charge density in the membrane. In the ISFET registration, this means that the static output voltage of the ISFET amplifier, determined by the $\mathrm{pH}$ of the electrolyte, shows a temporary disturbance with an amplitude which is related to the fixed charge density in the membrane. Because this charge density is a function of the $\mathrm{pH}$ of the electrolyte, the isoelectric point of the proteins can also be determined by gradually changing the electrolyte $\mathrm{pH}$, as shown in figure 10 .

Similarly, the difference in isoelectric points of an antibody (or antigen) immobilised in the ISFET membrane and the

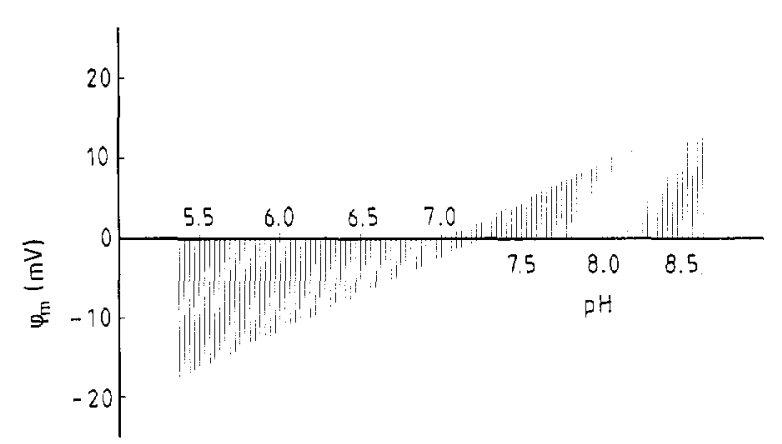

Figure 10. Sequence of recorded transient potentials as a function of $\mathrm{pH}$ for a lysozyme-loaded membrane of cross-linked polystyrene beads. 
antibody/antigen complex after an immunoreaction can be determined.

Although the measuring procedure should be evaluated to achieve a practically usable IMFET for the direct monitoring of an immunological reaction, it will be clear at this point that the method is based on a non-equilibrium phenomenon. The ISFET is used to measure induced transient membrane potentials, which occur within seconds, preventing any problem with ISFET drift.

\section{Conclusions}

As described in $\S 1$, the ISFET research and development efforts have, historically, been determined by comparison with the glass-membrane electrode, both theoretically and with respect to their applications, where the ISFET was seen as an alternative with a solid-state performance and of microdimensions. A serious drawback was encountered in the longterm drift, which is considerable compared with the drift of glass-membrane electrodes. Therefore ISFETS have been applied recently in cases where the fast response and the small dimensions are fully exploited in measuring methods where the drift behaviour is of minor importance.

\section{Acknowledgement}

This paper could not have been written without the continuous enthusiastic, critical and encouraging discussions with my scientific staff members and technicians as well as the $\mathrm{PhD}$ and MSc students.

\section{References}

[1] Bousse L, de Rooy N F and Bergveld P 1983 Operation of chemically sensitive field-effect sensors as a function of the insulator-electrolyte interface IEEE Trans. Electron Devices ED-30 1263-70

[2] Fung C D, Cheung P W and Ko W H 1986 A generalized theory of an electrolyte-insulator semiconductor field-effect transistor IEEE Trans. Electron Devices ED-33 8-18

[3] Baucke F G K 1985 The glass electrode - applied electrochemistry of glass surfaces J. Non-Cryst. Solids 73 215-31

[4] Bergveld P 1986 The development and application of FET-based biosensors Biosensors 2 15-33

[5] Ligtenberg H C G 1987 Baseline drift mechanism of $\mathrm{SiO}_{2} / \mathrm{Al}_{2} \mathrm{O}_{3} \mathrm{pH}$ ISFETS and some improvements by pulsed excitation Proc. Transducers '87 (Japan) (Tokyo: IEE Japan) pp 747-50

[6] Van den Vlekkert H H 1988 Some fundamental and practical aspects of CHEMFETs Thesis University of Neuchâtel, Switzerland

[7] Sibbald A 1985 A chemical sensitive integrated circuit: the operational transducer Sens. Actuators 7 23-38

[8] Bergueld P, Van den Berg A, Van der Wal P D, Skowronska-Ptasinska M, Sudhölter E J R and Reinhoudt D N 1989 How electrical and chemical requirements for REFETS may coincide Sens. Actuators 18 307-25

[9] Sibbald A and Covington A K 1985 Online patientmonitoring system for the simultaneous analysis of blood $\mathrm{K}^{+}, \mathrm{Ca}^{2+}, \mathrm{Na}^{+}$and $\mathrm{pH}$ using a quadruplefunction ChemfET integrated circuit sensor Med. Biol. Eng. Comp. 23 329-38
[10] Gumbrecht W, Schelter W and Montag B 1987 A chemFET microcell system for medical and biotechnological online electrolyte monitoring Tech. Digest Eurosensors I (Cambridge) pp 155-6

[11] Tamura T, Togawa T, Suematsu K and Sato K 1989 Continuous blood $\mathrm{pH}$ monitoring by use of the null method Sens. Actuators 16 273-85

[12] Van der Schoot B H and Bergveld P 1985 An Isferbased microlitre titrator: integration of a chemical sensor-actuator system Sens. Actuators 8 11-22

[13] Van der Schoot B H and Bergveld P 1988 Coulometric sensors, the application of a sensor-actuator system for long-term stability in chemical sensing Sens. Actuators 13 251-62

[14] Olthuis W, Van der Schoot B H, Chavez F and Bergueld P 1989 A dipstick sensor for coulometric acid-base titrations Sens. Actuators 17 279-84

[15] Schasfoort R B M, Kooyman R P H, Bergveld P and Greve J 1989 Modulation of ISFET response by an immunological reaction Sens. Actuators 17 531-5 